\title{
Congenital myotonia: a review of twenty cases and a new splice-site mutation in the CLCN1 gene
}

\author{
Nezir Özgün ${ }^{1 \oplus}$, Hasan Taşlıdere ${ }^{2 \odot}$ \\ ${ }^{1}$ Department of Pediatrics, Division of Pediatric Neurology, İstinye University School of Medicine, İstanbul; ${ }^{2}$ Department of Medical \\ Genetics, University of Health Sciences, Haseki Training and Research Hospital, İstanbul, Turkey.
}

\begin{abstract}
Background and objectives. Congenital Myotonia (CM) is a disease caused by mutations in the skeletal muscle chloride channel gene (CLCN1). Mutations can be transmitted as autosomal dominant (Thomsen's disease) or recessive (Becker's disease). CM is more common in men and Becker myotonia may be 10 times more common than Thomsen myotonia. Genotypic and phenotypic characteristics of CM may vary according to geographical region and ethnicity.
\end{abstract}

Method. In this study, we present the genotypic and phenotypic characteristics of 20 Turkish CM patients all diagnosed by molecular genetic testing.

The clinical and laboratory features of the patients with mutation in CLCN1 gene were retrospectively analyzed.

Results. Eleven of the patients were female. c.1064+1G>A splice-site change, p.Arg338X (c.1012 C>T) stop codon, p.Gly190Ser (c.568_569delinsTC) missense mutations were detected. Eight of the 20 patients were found to be compatible with Becker type and 12 with Thomsen type, based on mode of inheritance, neurological examination findings and genetic test results.

Conclusion. The c.1064+1G>A splice-site change mutation, defined for the first time in this study, expands the spectrum of mutations in the CLCN1 gene. Thomsen type and female gender were observed to be more frequent in this series of patients from Turkey.

Key words: Congenital myotonia, Thomsen disease, Becker disease, CLCN1 gene.

Congenital Myotonia (CM) is a disease caused by mutations in the skeletal muscle chloride channel gene (CLCN1). ${ }^{1}$ The sarcolemmal chloride conductance is reduced due to the defect in the CLCN1 gene encoding the ClC1 chloride channel, resulting in both clinically and also electrically delayed relaxation of the hyperpolarized muscle. Affected patients describe muscle stiffness after a strong contraction. $^{2-4}$ The mutation in the CLCN1 gene is divided into two subgroups according to mode

$\triangle$ Nezir Özgün

nezirozgun@hotmail.com

Received 12th November 2019, revised 20th February 2020, accepted 3rd April 2020.

This was presented at the 3rd Neuromuscular Diseases Congress, 1-3 November 2019, Çeşme, İzmir, Turkey. of inheritance: autosomal dominant (Thomsen disease, OMIM\#160800) and autosomal recessive (Becker disease, OMIM\#255700). ${ }^{5}$ Genotype-phenotype correlations are variable and various intermediate forms have been described between Thomsen and Becker phenotypes. ${ }^{6}$ Thomsen and Becker forms can be distinguished by time of onset, mode of inheritance, and clinical findings. Clinical and genetic characteristics of these two forms are summarized in Table I. , $^{, 5,7-13}$

CM was reported to be more common in men.,14 The incidence was initially estimated as 1:23.000 for the Thomsen type and 1:50.000 for the Becker type ${ }^{15}$; in subsequent studies, however, Becker type was reported to be more common. ${ }^{16}$ The prevalence of CM was estimated to be 1:10.000 
Table I. Clinical and genetic characteristics of Thomsen and Becker myotonia.

\begin{tabular}{lcc}
\hline & Thomsen & Becker \\
\hline $\begin{array}{l}\text { Gene and gene location } \\
\text { Inheritance type }\end{array}$ & CLCN1 / 7q35 & CLCN1 / 7q35 \\
Onset age & Autosomal Dominant & Autosomal Recessive \\
Male/Female Ratio & At any age & Early Childhood \\
Myotonia Severity & 1.3 & 2.0 \\
Muscle Hypertrophy & From asymptomatic to severe & Usually severe \\
Episodic weakness & Rare, light and generalized & More frequent and mostly in the \\
Permanent weakness & None & lower extremities \\
Increased findings over the years & None & Rare \\
\hline
\end{tabular}

in Northern Scandinavia ${ }^{17,18}$, while 1-10: 100,000 worldwide. ${ }^{19}$

Genotypic and phenotypic characteristics of CM may vary according to geographical region and ethnicity. ${ }^{6,7,14-18}$ There are limited number of studies on CM in Turkey, which are mostly related to neurophysiological characteristics of the disease and not diagnosed by molecular genetic testing at all cases..$^{20-22}$ There are no studies reporting genotypic and phenotypic characteristics of patients.

In this study we present the genotypic and phenotypic characteristics of $20 \mathrm{CM}$ patients from three Turkish families, all diagnosed by molecular genetic testing. We determined three different mutations and one of these is a novel mutation identified for the first time in the CLCN1 gene.

\section{Material and Methods}

Analysis of mutations in CLCN1 gene was performed in 5 index cases from three families who applied to the pediatric neurology outpatient clinic of our hospital with the symptoms suggesting nondystrophic $\mathrm{CM}$, in the light of clinical examination and laboratory findings. Upon mutation-positive test results, family screening was performed. Mutational analysis in the CLCN1 gene was performed in 24 individuals from three families, including parents, children and one aunt in the first family (Fig. 1). The clinical and laboratory features of the patients with mutation were retrospectively analyzed. The mode of inheritance was determined according to the neurological examination findings and genetic results. Consent form was obtained after providing a detailed information from all patients or their legal guardians. The study was conducted in accordance with the rules of the Helsinki Declaration of Ethics. We received ethics committee approval-Diyarbakır Gazi Yaşargil Training and Research Hospital (Number: 356/25.10.2019).

\section{Myotonia Assessment}

Neurological examination was performed to search for myotonic findings, particularly in the tongue, jaw, eyelid and limbs. Handgrip myotonia, being unable to open the eyes after a strong eyelid closure and percussion myotonia were examined. The semi-quantitative scale of myotonic impairment ${ }^{15}$ was used to grade the severity of myotonia. Readily noticeable myotonia hindering daily life activities was defined as severe myotonia (+++). The case is defined as moderate myotonia (++) if some minor troubles in daily life can be noticed and reported by the individuals, but it is called as mild myotonia (+) in case it has no noticable negative effects on daily life but can be diagnosed only by examination. 

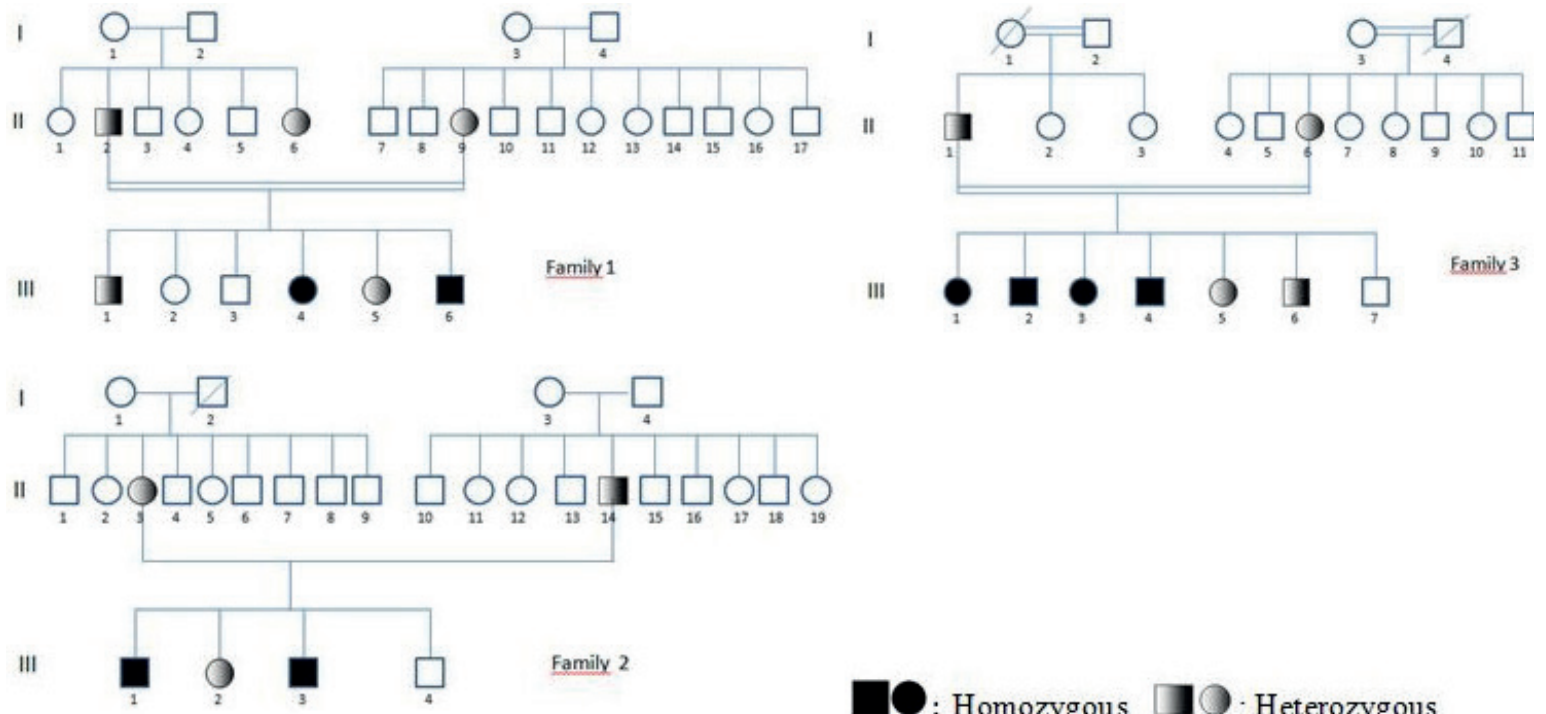

$\square$ : Homozygous $\square$ : Heterozygous

Fig. 1. Pedigree of families.

\section{Genetic Diagnosis}

Exons 1-23 of the CLCN1 gene were amplified by polymerase chain reaction (PCR) and then DNA sequencing was performed. DNA isolation from peripheral blood samples was performed using saline precipitation method. Exons of the CLCN1 gene obtained from isolated DNAs were amplified by PCR. Amplification was confirmed by agarose gel electrophoresis. Illumina's Nextera XT Library Prep Kit was used for making sequencing libraries from the amplified exon regions for next generation sequencing. Purification was then performed using AMPure $\mathrm{XP}$ kit (Beckman Coulter) and DNA libraries were loaded onto Illumina Miseq System using MiSeq v2 Reagent kits. The BAM files provided by Miseq instrument were viewed and analyzed by IGV program. Cases with mutations were confirmed by Sanger sequencing.

\section{Results}

\section{General Clinical Characteristics}

Mutations were detected in 20 cases. Eleven of the patients $(55 \%)$ were female. The parents had first-degree kinship relation in the first and third families. Difficulty in opening hands, worsening in cold weather and palliating after repetitive contractions, difficulty in motility and fatigue were common complaints of the patients who were diagnosed to have myotonia in neurological examination. Developmental phases such as sitting, crawling and walking were normal in all patients with mutation. Complaints and clinical findings of all patients with myotonia began within the first 3 years. All patients, either heterozygous or homozygous, were able to perform daily life activities independently without any support. Homozygous mutation was detected in each index patient. Muscle hypertrophy was determined only in patients with homozygous transmission in addition to myotonia, in their neurological examination. Mild hypertrophy was determined in the members of the first and second families especially localized in the lower extremities, while generalized and marked muscular hypertrophy (Hercules' appearance) in all family members with homozygous mutations in the third family (Fig. 2). Electromyographic examination (EMG) was done only in one person, the index case, from each family, considering that it is a painful procedure. Motor unit potentials were normal but myotonic discharges were observed. Clinical findings remained unchanged in the first and second families while increased in the third family over the years. 


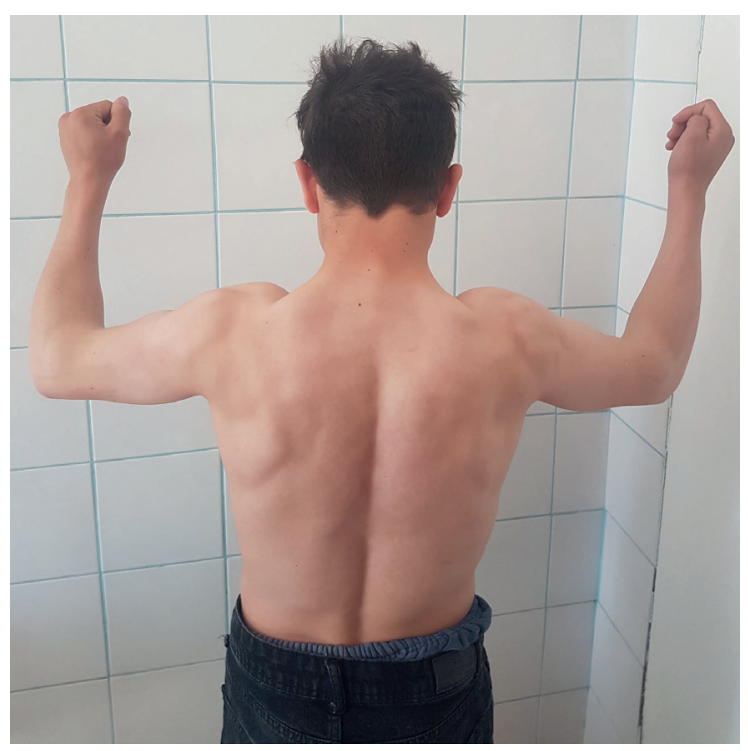

Fig. 2. Example of generalized muscle hypertrophy and Hercules' appearance in the patients with homozygous p.Gly190Ser mutation.

Demographic, clinical, genetic, and laboratory characteristics of patients with mutation and the grade that the patient was affected by myotonia are summarized in Table II. Eight (40\%) of the 20 patients were found to be compatible with Becker type and 12 (60\%) with Thomsen type, based on mode of inheritance, neurological examination findings and genetic test results.

\section{Genetic Test Results}

In the first family, c.1064+1G>A splice-site change mutation was detected in intron 9 of the CLCN1 gene. The mutation was confirmed by Sanger sequencing. Sanger sequencing image of the detected mutation is shown in Figure 3.
The mutation was homozygous in two index siblings and heterozygous in all other family members.

In the second family, p.Arg338X (c.1012 C>T) stop codon mutation was detected in exon 9 of the CLCN1 gene. The mutation was homozygous in index cases and heterozygous in sisters and parents. No mutation was detected in six-year-old brother.

In the third family, p.Gly190Ser (c.568_569delinsTC) missense mutation was detected in the exon 5 of the CLCN1 gene. The mutation was homozygous in the four oldest children and heterozygous in the other family members. No mutation was detected in the twoyear-old brother.

\section{Treatment}

Treatment was not needed in the patients with the Thomsen variant because myotonia was mild and causing no difficulties in daily life. Treatment was initiated for the patients in all three families diagnosed as severe myotonia based on the semi-quantitative scale of myotonic impairment and suffering difficulties in daily life activities such as walking, going up the stairs and writing. Primarily carbamazepine was initially given to the patients of the first and second families, while directly mexiletine was prescribed to the patients of the third family. Mexiletine was initiated when carbamazepine failed to resolve the complaints significantly, even at increased doses of $20 \mathrm{mg} / \mathrm{kg} /$ day. In all patients with severe myotonia according to the

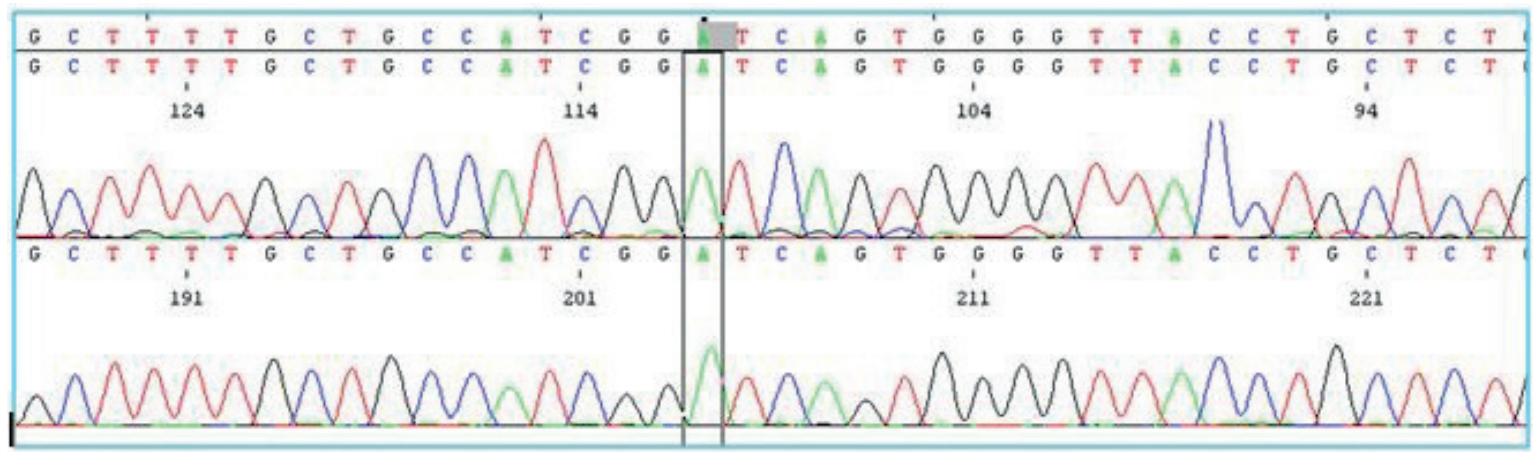

Fig. 3. Sanger sequencing image of c.1064+1G>A splice-site change mutation. 


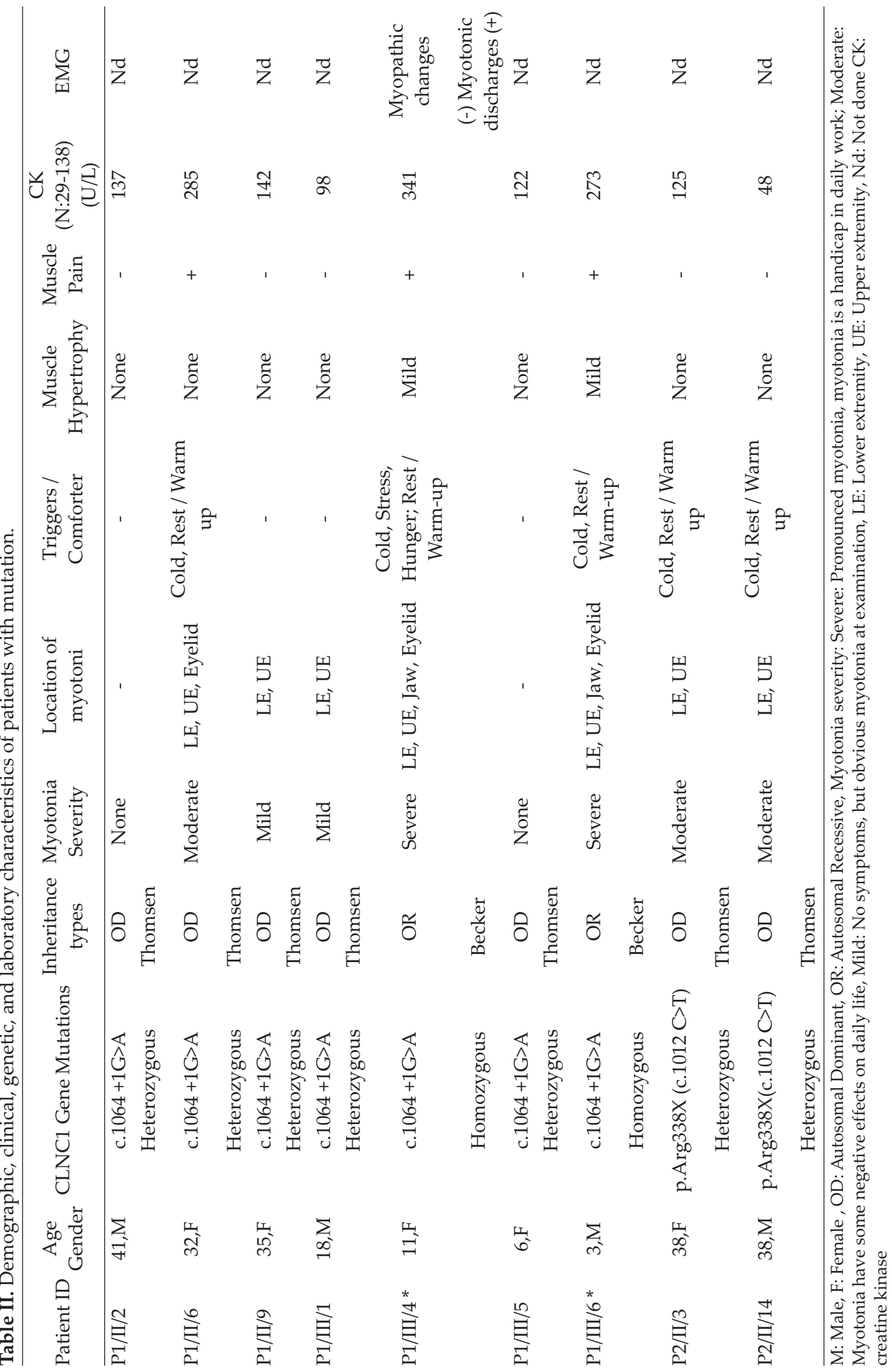




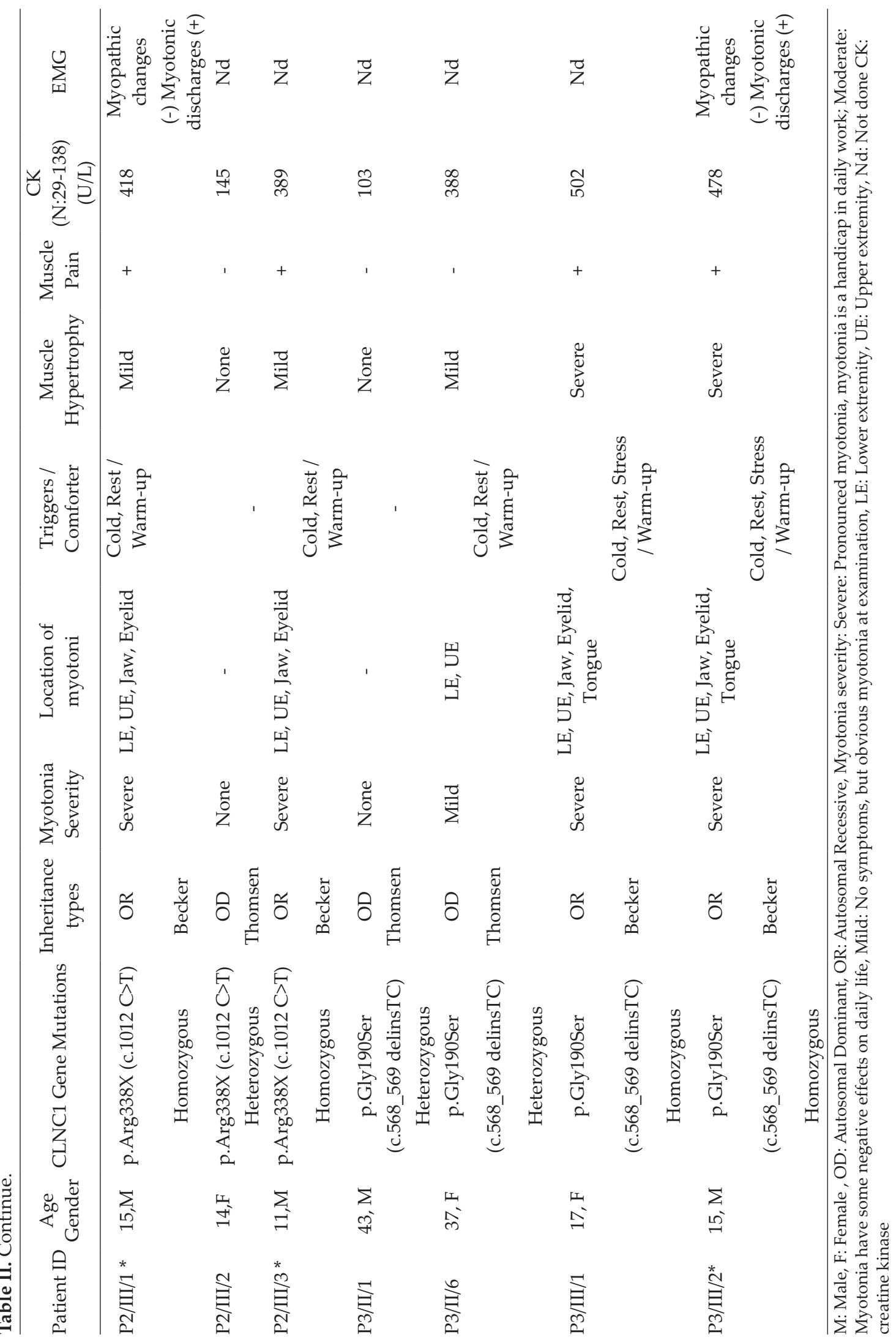




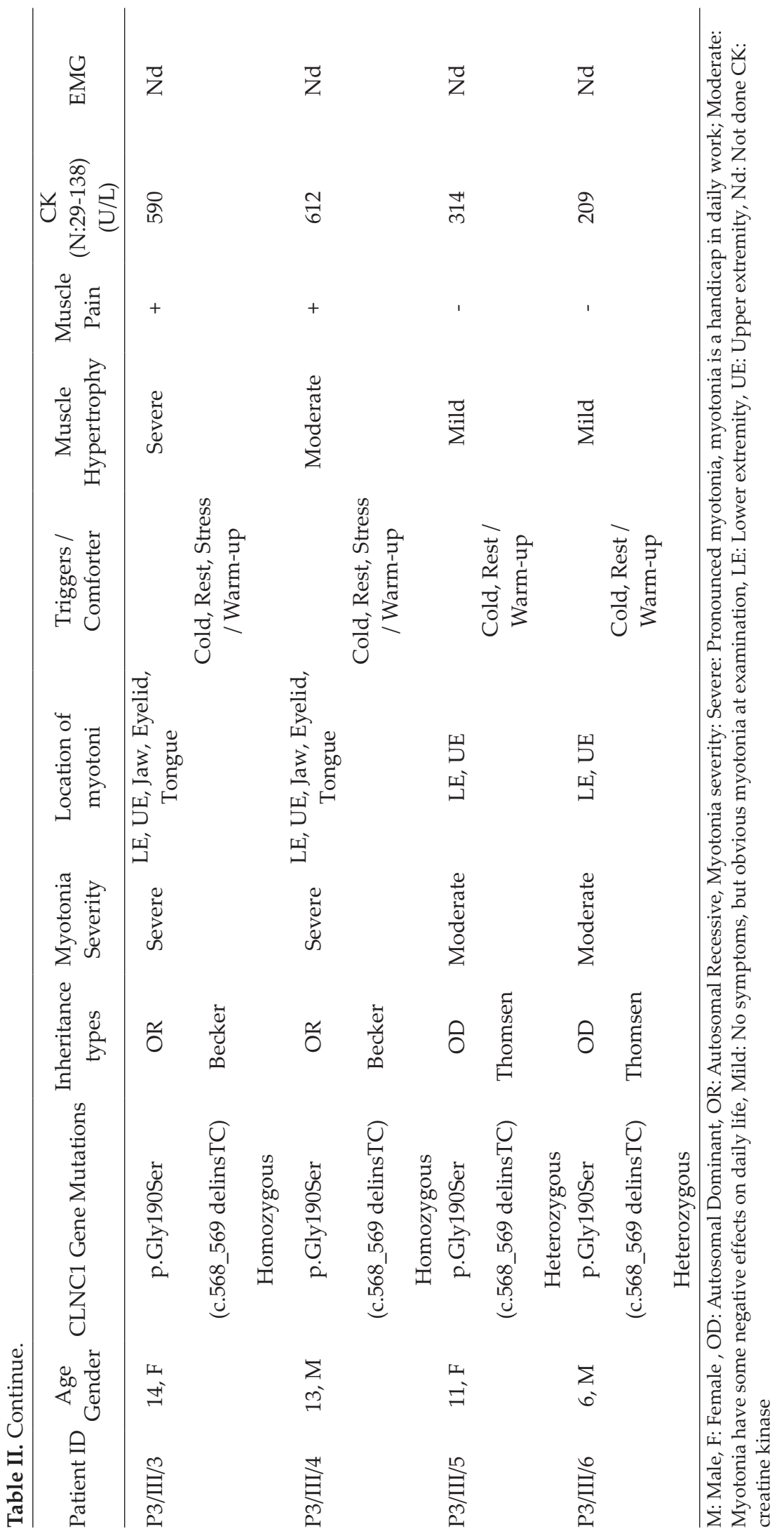


semi-quantitative scale of myotonic impairment and given mexiletine $(2 \times 150 \mathrm{mg})$, the severity of myotonia regressed to moderate and on some of the days to mild.

\section{Discussion}

In this study, phenotypic and genotypic characteristics of Turkey's series of CM cases are presented, mostly consisting of pediatric patients and verified by molecular genetic testing.

The first mutations in CLCN1, both recessive and dominant, have been reported in the early 1990s ${ }^{1}$, and more than 200 pathogenic mutations were identified so far..$^{23}$ Myotoniainducing mutations are spread throughout the entire sequence of the channel protein and are mostly seen as deletion, insertion, duplication, frame shift, stop codon, 'missense' or 'splicesite' mutations. ${ }^{11,23}$ In $\mathrm{CM}$, the same mutation may show both homozygous and heterozygous transition. Heterozygous mutations are usually asymptomatic, but marked myotonia may occur without symptoms of weakness and myopathy. There may be significant phenotypic differences even among family members carrying the same mutation. ${ }^{4,24-26}$ This phenotypic difference may be due to variable expression, incomplete penetrance, the effect of mutant alleles on wild type channel proteins, allele expression, gene dose effect and specific variability of channel dysfunction. ${ }^{27,28}$ Clinical and electrophysiological findings may be sufficient to establish the diagnosis, but do not allow any distinction between recessive and dominant forms. It is difficult to evaluate heredity and genotype-phenotype correlation without examining the patient and pedigree, even if they have the same mutation. Only the father and six years old girl were asymptomatic while the other 4 members of the family with heterozygous transition of c.1064+1G $>$ A mutation, that we identified the first time, had mild to moderate myotonia. Severe myotonia and mild muscle hypertrophy in the lower extremities were determined in those patients with homozygous transition. Clinical findings were generally consistent with the literature. These results allow us to expand the spectrum of CLCN1 alleles as well as verify that the c.1064+1G>A splice-site change mutation is both heterozygous and homozygous dominant mutation with full penetration.

The p.Arg338X (c.1012 C>T) stop codon mutation detected in the second family and the p.Gly190Ser missense mutation detected in the third family were previously identified in the literature. ${ }^{29,30}$ Also these mutations displayed both homozygous and heterozygous transition. Disease onset age, heat sensitivity, severity of myotonia and distribution characteristics of our patients with p.Arg338X (c.1012 C>T) stop codon mutation were similar to the clinical features of the patient described in detail by Ulzi et al. ${ }^{29}$ In our patients however, those with homozygous mutation had mild muscle hypertrophy, while their patient had no hypertrophy but hypotrophy. This difference may be related to the p.Gly190Ser mutation besides the p.Arg338X mutation of the patient they identified, or to some other factors.

The p.Gly190Ser missense mutation detected in the third family was first described by Shalata et al. ${ }^{30}$ in 12 members of an Arab family. Although the father had a heterozygous mutation, he had had no symptoms or complaints, and he even had successful results in athletics competitions during his military service. Generalized muscle hypertrophy and Hercules' appearance were determined in the patients with homozygous p.Gly190Ser mutation, unlike mild muscle hypertrophy observed in the lower extremities of the other two mutations of our cases, in the homozygous variants. The phenotype of our both heterozygous and homozygous patients was quite similar to the patient series reported by Shalata et al. ${ }^{30}$

$\mathrm{CM}$ is the most common hereditary skeletal muscle canalopathy. ${ }^{13,15}$ Becker myotonia may be 10 times more common than Thomsen myotonia. ${ }^{6}$ There are very few reports published on Thomsen myotonia. In a French-Canadian 
cohort of 50 patients, 27 patients were reported as Becker type and 9 patients as Thomsen type. ${ }^{31}$ Another study conducted with 142 families from West Germany, reported that 73\% of the cases were Becker type while $19 \%$ were Thomsen type. ${ }^{25}$ In our series of 20 patients, clinical and genetic transmission compatible with Thomsen myotonia was determined in $12(60 \%)$ and with Becker myotonia in 8 (40\%) patients. In a study from England with patients that were genetically proven to have skeletal muscle canalopathy, 99 patients had Thomsen and 69 had Becker variants and two types were observed in similar rates. ${ }^{13}$ Again, $86.1 \%$ of the cases reported from China were reported to be Thomsen variants. ${ }^{32}$ The findings in our series as well as in the other studies suggest that the incidence of the two types vary in accordance with ethnicity and regions. CM was reported to be seen nearly twice in males than in females, ${ }^{7,14}$, but female $(55 \%)$ and male $(45 \%)$ rates were almost equal in our patient series.

Electromyography (EMG), by displaying myotonic discharges, is useful in demonstrating myotonic disorder and in distinguishing CM from paramyotonia congenita or periodic paralysis, but not in distinguishing between dominant and recessive variants of CM. ${ }^{16,33}$ Sequence analysis of CLCN1 detects more than 95\% of CMpathogenic variants of both autosomal recessive and autosomal dominant forms. Deletion/duplication analysis in the CLCN1 gene detects the remaining $1-5 \%$ mutations. ${ }^{9}$ EMG was performed only in 3 patients, because clinical findings of our patients were consistent with nondystrophic CM. The other patients underwent only genetic examination. It may be in favor of patients' comfort to perform directly genetic examination, if the clinic view of the patient is compatible with nondystrophic CM.

Mexiletin, a derivative of lidocaine, is the most commonly used treatment option with proven efficacy in randomized controlled trials. ${ }^{34-36}$ Besides Mexiletine, various sodium channel blockers such as phenytoin and carbamazepine can be used. Besides these agents, lithium, tocainide, and trimeprazine have also been tested. $^{37}$ In two recent studies, ranolazine ${ }^{38}$ and lamotrigine ${ }^{39}$ were suggested to be used in nondystrophic CM. Lamotrigine was reported to be considered as a cheaper and effective first choice because of the high cost and side effects of Mexiletin..$^{39}$ We could not obtain an adequate clinical response despite prescribing up to 20 $\mathrm{mg} / \mathrm{kg} /$ day doses of carbamazepine, although Lyons et al..$^{40}$ noted a dramatic response to lowdose carbamazepine in patients with Becker variant. Based on the semiquantitative scale of myotonic impairment, a significant decrease was determined in the severity of myotonia in all patients that started mexiletine after stopping carbamazepine.

In conclusion, the c.1064+1G>A splice-site change mutation, defined for the first time in this study, expands the spectrum of mutations in the CLCN1 gene and contributes to genotypephenotype correlation. Although Becker type and the male gender were reported to be more common in the literature so far, Thomsen type and female gender were observed to be more frequent in this series of patients from Turkey, which was also confirmed genetically.

\section{Acknowledgment}

We would like to thank Dr. Haluk Topaloğlu and Dr. Uluç Yiş for their valuable criticisms and contributions.

\section{REFERENCES}

1. Koch MC, Steinmeyer K, Lorenz C, et al. The skeletal muscle chloride channel in dominant and recessive human myotonia. Science 1992; 257: 797-800.

2. Pusch M. Myotonia caused by mutations in the muscle chloride channel gene CLCN1. Hum Mutat 2002; 19: 423-434.

3. Pusch M, Steinmeyer K, Koch MC, Jentsch TJ. Mutations in dominant human myotonia congenita drastically alter the voltage dependence of the CIC-1 chloride channel. Neuron 1995; 15: 1455-1463.

4. Lakraj AA, Miller G, Vortmeyer AO, Khokhar B, Nowak RJ, DiCapua DB. Novel mutations in the ClCN-1 gene of myotonia congenita: 2 case reports. Yale J Biol Med 2013; 86: 101-106. 
5. Matthews E, Fialho D, Tan SV, et al; $\mathrm{CINCH}$ Investigators. The non-dystrophic myotonias: molecular pathogenesis, diagnosis and treatment. Brain 2010; 133(Pt 1): 9-22.

6. Derevenciuc A, Abicht A, Hamza S, Roth C, Ferbert A. Thomsen myotonia-A 4-generation family with a new mutation and a mild phenotype. Muscle Nerve 2016; 53: 653-654.

7. Mailänder V, Heine R, Deymeer F, Lehmann-Horn F. Novel muscle chloride channel mutations and their effects on heterozygous carriers. Am J Hum Genet 1996; 58: 317-324.

8. Imbrici P, Maggi L, Mangiatordi GF, et al. ClC-1 mutations in myotonia congenita patients: insights into molecular gating mechanisms and genotypephenotype correlation. J Physiol 2015; 593: 41814199.

9. Dunø M, Colding-Jørgensen E. Myotonia congenita. In: Adam MP, Ardinger HH, Pagon RA, Wallace SE, Bean LJH, Stephens K, Amemiya A (eds). GeneReviews. Seattle WA: University of Washington, 1993-2020. 2005 Aug 3 [updated 2015 Aug 6].

10. Hahn C, Salajegheh MK. Myotonic disorders: a review article. Iran J Neurol 2016; 15: 46-53.

11. Brugnoni R, Kapetis D, Imbrici P, et al. A large cohort of myotonia congenita probands: novel mutations and a high-frequency mutation region in exons 4 and 5 of the CLCN1 gene. J Hum Genet 2013; 58: 581-587.

12. Kato H, Kokunai $Y$, Dalle $C$, et al. A case of nondystrophic myotonia with concomitant mutations in the SCN4A and CLCN1 genes. J Neurol Sci 2016; 369: 254-258.

13. Horga A, Raja Rayan DL, Matthews E, et al. Prevalence study of genetically defined skeletal muscle channelopathies in England. Neurology 2013; 80: 1472-1475.

14. Fialho D, Kullman DM, Hanna MG, Schorge S. Non-genomic effects of sex hormones on ClC-1 may contribute to gender differences in myotonia congenita. Neuromuscul Disord 2008; 18: 869-872.

15. Becker PE. Myotonia congenita and syndromes associated with myotonia. In: Becker PE, Lenz W, Vogel F, Wendt GG (eds). Vol. 3, Stuttgard, Germany: Georg Thieme Verlag, 1977.

16. Fialho D, Schorge S, Pucovska U, et al. Chloride channel myotonia: exon 8 hot-spot for dominantnegative interactions. Brain 2007; 12: 3265-3274.

17. Papponen H, Toppinen T, Baumann $P$, et al. Founder mutations and the high prevalence of myotonia congenita in northern Finland. Neurology 1999; 53: 297-302.
18. Sun C, Tranebjaerg L, Torbergsen T, Holmgren G, Van Ghelue M. Spectrum of CLCN1 mutations in patients with myotonia congenita in Northern Scandinavia. Eur J Hum Genet 2001; 9: 903-909.

19. Emery AE. Population frequencies of inherited neuromuscular diseases - a world survey. Neuromuscul Disord 1991; 1: 19-29.

20. Deymeer F, Cakirkaya S, Serdaroğlu P, et al. Transient weakness and compound muscle action potential decrement in myotonia congenita. Muscle Nerve 1998; 21: 1334-1337.

21. Deymeer F, Lehmann-Horn F, Serdaroğlu P, et al. Electrical myotonia in heterozygous carriers of recessive myotonia congenita. Muscle Nerve 1999; 22: $123-125$.

22. Sahin I, Erdem HB, Tan H, Tatar A. Becker's myotonia: novel mutations and clinical variability in patients born to consanguineous parents. Acta Neurol Belg 2018; 118: 567-572.

23. Coote DJ, Davis MR, Cabrera M, Needham M, Laing NG, Nowak KJ. Clinical utility gene card for: autosomal dominant myotonia congenita (Thomsen disease). Eur J Hum Genet 2018; 26: 1072-1077.

24. Cassone M, Ferradini V, Longo G, et al. Genotypephenotype correlation of F484L mutation in three Italian families with Thomsen myotonia. Muscle Nerve 2017; 55: E24-E25.

25. Colding-Jørgensen E. Phenotypic variability in myotonia congenita. Muscle Nerve 2005; 32: 19-34.

26. Gurgel-Giannetti J, Senkevics AS, ZilbersztajnGotlieb D, et al. Thomsen or Becker myotonia? A novel autosomal recessive nonsense mutation in the CLCN1 gene associated with a mild phenotype. Muscle Nerve 2012; 45: 279-283.

27. Duno M, Colding-Jorgensen E, Grunnet M, Jespersen T, Vissing J, Schwartz M. Difference in allelic expression of the CLCN1 gene and the possible influence on the myotonia congenita phenotype. Eur J Hum Genet 2004; 12: 738-743.

28. Kumar KR, Ng K, Vandebona H, Davis MR, Sue CM. A novel CLCN1 mutation (G1652A) causing a mild phenotype of thomsen disease. Muscle Nerve 2010; 41: 412-415.

29. Ulzi G, Lecchi M, Sansone V, et al. Myotonia congenita: novel mutations in CLCN1 gene and functional characterizations in Italian patients. J Neurol Sci 2012; 318: 65-71.

30. Shalata A, Furman H, Adir V, et al. Myotonia congenita in a large consanguineous Arab family: insight into the clinical spectrum of carriers and double heterozygotes of a novel mutation in the chloride channel CLCN1 gene. Muscle Nerve 2010; 41: 464-469. 
31. Dupré N, Chrestian N, Bouchard JP, et al. Clinical, electrophysiologic, and genetic study of nondystrophic myotonia in French-Canadians. Neuromuscul Disord 2009; 19: 330-334.

32. Gao F, Ma FC, Yuan ZF, et al. Novel chloride channel gene mutations in two unrelated Chinese families with myotonia congenita. Neurol India 2010; 58: 743-746.

33. Fournier E, Arzel M, Sternberg D, et al. Electromyography guides toward subgroups of mutations in muscle channelopathies. Ann Neurol 2004; 56: 650-661.

34. Ørstavik K, Wallace SC, Torbergsen T, et al. A de novo mutation in the SCN4A gene causing sodium channel myotonia. J Neuromuscul Dis 2015; 2: 181184.

35. Ginanneschi F, Mignarri A, Lucchiari S, et al. Neuromuscular excitability changes produced by sustained voluntary contraction and response to mexiletine in myotonia congenita. Neurophysiol Clin 2017; 47: 247-252.
36. Statland JM, Bundy BN, Wang $\mathrm{Y}$, et al; and the Consortium for Clinical Investigation of Neurologic Channelopathies. Mexiletine for symptoms and signs of myotonia in non-dystrophic myotonia: a randomized controlled trial. JAMA 2012; 308: 1357 1365.

37. Conravey A, Santana-Gould L. Myotonia congenita and myotonic dystrophy: surveillance and management. Curr Treat Options Neurol 2010; 12: 16-28.

38. Arnold WD, Kline D, Sanderson A, et al. Open-label trial of ranolazine for the treatment of myotonia congenita. Neurology 2017; 89: 710-713.

39. Andersen G, Hedermann G, Witting N, Duno M, Andersen $\mathrm{H}$, Vissing J. The antimyotonic effect of lamotrigine in non-dystrophic myotonias: a doubleblind randomized study. Brain 2017; 140: 2295-2305.

40. Lyons MJ, Duron R, Molinero I, Sangiuolo F, Holden KR. Novel CLCN1 mutation in carbamazepineresponsive myotonia congenita. Pediatr Neurol 2010; 42: 365-368. 